\title{
God plays coins or superposition principle for quantum states in probability representation of quantum mechanics
}

\author{
Olga Man'ko ${ }^{1,2,{ }^{*}}$ and Vladimir Chernega ${ }^{1}$ \\ ${ }^{1}$ Lebedev Physical Institute, Russian Academy of Sciences, 119991 Moscow, Russia \\ ${ }^{2}$ Bauman Moscow State Technical University, 105005 Moscow, Russia
}

\begin{abstract}
The superposition principle of quantum states is expressed as the addition rule of probability distributions which are identified with these quantum states in new representation of quantum mechanics. Example of two spin- $1 / 2$ states is considered explicitly.
\end{abstract}

Recently new representation of quantum mechanics was considered [1]-[4]. In this representation the probability distributions describe the quantum states and these distributions determine the wave functions (for pure states) and density matrices (for mixed states). The fundamental principle of quantum mechanics is the superposition principle which is expressed as addition rule of wave functions or state vectors $\left|\psi_{1}\right\rangle$ and $\left|\psi_{2}\right\rangle$ with complex coefficients, i.e. $\left|\psi>=c_{1}\right| \psi_{1}>+c_{2} \mid \psi_{2}>$. The obtained state vector $|\psi\rangle$ describes another state of the system. The corresponding density operators $\widehat{Q_{1}}=\left|\psi_{1}><\psi_{1}\right|$ and $\widehat{\varrho_{2}}=\left|\psi_{2}><\psi_{2}\right|$ and their density matrices are expressed in probability representation of quantum mechanics in terms of dichotomic probabilities [5]. For example the spin-1/2 density matrix and spin-1 density matrix are given in the form

and

$$
\varrho^{\left(\frac{1}{2}\right)}=\left(\begin{array}{cc}
p_{3} & p_{1}-\frac{1}{2}-i\left(p_{2}-1 / 2\right) \\
p_{1}-\frac{1}{2}+i\left(p_{2}-1 / 2\right) & 1-p_{3}
\end{array}\right)
$$

$$
=\left(\begin{array}{ccc}
p_{3}^{(11)} & p_{1}^{(12)}-\frac{1}{2}-i\left(p_{2}^{(12)}-1 / 2\right) & p_{1}^{(13)}-\frac{1}{2}-i\left(p_{2}^{(13)}-1 / 2\right) \\
p_{1}^{(12)}-\frac{1}{2}+i\left(p_{2}^{(12)}-1 / 2\right) & p_{3}^{(22)} & p_{1}^{(23)}-\frac{1}{2}-i\left(p_{2}^{(23)}-1 / 2\right) \\
p_{1}^{(13)}-\frac{1}{2}+i\left(p_{2}^{(13)}-1 / 2\right) & p_{1}^{(23)}-\frac{1}{2}+i\left(p_{2}^{(23)}-1 / 2\right) & 1-p_{3}^{(11)}-p_{3}^{(22)}
\end{array}\right)
$$

respectively. The numbers $0 \leq p_{1}, p_{2}, p_{3} \leq 1$ are the probabilities of spin-1/2 projections $\mathrm{m}=+1 / 2$ on the axes $\mathrm{x}, \mathrm{y}, \mathrm{z}$ in the given state with density matrix $\varrho^{\left(\frac{1}{2}\right)}$. Analogously the numbers $0 \leq p_{1,2,3}^{\left(j, k^{k}\right)} \leq 1, j, k=1,2,3$ and $\mathrm{j}<\mathrm{k}$ are probabilities for artificial spin- $1 / 2$ systems

\footnotetext{
* Corresponding author: mankoov@lebedev.ru
} 
determined by the spin-1 system. This property is expressed as extra inequality for the probabilities given by Silvester criterion. The superposition principle is expressed in terms of density operators (density matrices) [6] in the form

$$
\widehat{\varrho_{\psi}}=\sqrt{\lambda_{1}} \varrho_{1}+\sqrt{\lambda_{2}} \varrho_{2}+\sqrt{\lambda_{1} \lambda_{2}} \frac{\widehat{\varrho_{1} \varrho_{0} \varrho_{2}}+\widehat{\varrho_{2} \varrho_{0} \varrho_{1}}}{\sqrt{\text { Tr } \widehat{\varrho_{1}} \widehat{\varrho_{0}} \widehat{\varrho_{2} \varrho_{0}}}} .
$$

Here $\widehat{\varrho_{\psi}}=|\psi\rangle<\psi \mid, 0 \leq \lambda_{1}, \lambda_{2} \leq 1$ are probabilities $\lambda_{1}+\lambda_{2}=1$ and $\widehat{\varrho_{0}}=\left|\psi_{0}\right\rangle\left\langle\psi_{0}\right|$. Using this relation one can express matrix elements of density matrix $\widehat{\varrho}_{\psi}$ in terms of probabilities. For spin-1/2 state it gives the addition rule for the probabilities $\wp_{1}^{(1)}, \wp_{2}^{(1)}$, $\wp_{3}^{(1)}, \wp_{1}^{(2)}, \wp_{2}^{(2)}, \wp_{3}^{(2)}$, which gives the probabilities $\wp_{1}^{(\psi)}, \wp_{2}^{(\psi)}, \wp_{3}^{(\psi)}$ of the superposition state. We present the formula for the probabilities $\wp_{1}^{(\psi)}, \wp_{2}^{(\psi)}, \wp_{3}^{(\psi)}$ determining the superposition state $\widehat{\varrho_{\psi}}$. It reads [5] :

$$
\begin{aligned}
& \wp_{3}^{(\psi)}=\left(\frac{1}{\tau}\right)\left\{\wp_{3}^{(0)} \wp_{3}^{(1)}+\left(1-\wp_{3}^{(0)}\right) \wp_{3}^{(2)}+2 \sqrt{\wp_{3}^{(1)} \wp_{3}^{(2)}}\left(\wp_{1}^{(0)}-\frac{1}{2}\right)\right\} ; \\
& \wp_{1}^{(\psi)}-\frac{1}{2}=\left(\frac{1}{\tau}\right)\left\{\wp_{3}^{(0)}\left(\wp_{1}^{(1)}-\frac{1}{2}\right)+\left(\wp_{1}^{(2)}-\frac{1}{2}\right)\left(1-\wp_{3}^{(0)}\right)+\left[\left(\wp_{1}^{(0)}-\frac{1}{2}\right)\left(\wp_{1}^{(1)}-\frac{1}{2}\right)-\right.\right. \\
& \left.\left(\wp_{2}^{(0)}-\frac{1}{2}\right)\left(\wp_{2}^{(1)}-\frac{1}{2}\right)\right] \sqrt{\wp_{3}^{(2)} / \wp_{3}^{(1)}}+\left[\left(\wp_{1}^{(0)}-\frac{1}{2}\right)\left(\wp_{1}^{(2)}-\frac{1}{2}\right)-\left(\wp_{2}^{(0)}-\frac{1}{2}\right)\left(\wp_{2}^{(2)}-\right.\right. \\
& \left.\left.\frac{1}{2}\right)\right] \sqrt{\left.\wp_{3}^{(1)} / \wp_{3}^{(2)}\right\}}, \wp_{2}^{(\psi)}-\frac{1}{2}=\left(\frac{1}{\tau}\right)\left\{\wp_{3}^{(0)}\left(\wp_{2}^{(1)}-\frac{1}{2}\right)+\left(\wp_{2}^{(2)}-\frac{1}{2}\right)\left(1-\wp_{3}^{(0)}\right)+\right. \\
& {\left[\left(\wp_{1}^{(0)}-\frac{1}{2}\right)\left(\wp_{2}^{(1)}-\frac{1}{2}\right)-\left(\wp_{2}^{(0)}-\frac{1}{2}\right)\left(\wp_{1}^{(1)}-\frac{1}{2}\right)\right] \sqrt{\wp_{3}^{(2)} / \wp_{3}^{(1)}}+\left[\left(\wp_{2}^{(0)}-\frac{1}{2}\right)\left(\wp_{1}^{(2)}-\frac{1}{2}\right)-\right.} \\
& \left.\left(\wp_{1}^{(0)}-\frac{1}{2}\right)\left(\wp_{2}^{(2)}-\frac{1}{2}\right)\right] \sqrt{\left.\wp_{3}^{(1)} / \wp_{3}^{(2)}\right\}},
\end{aligned}
$$

where

$$
\begin{aligned}
& \tau=1+2\left\{\left(\wp_{1}^{(0)}-\frac{1}{2}\right)\left[\left(\wp_{1}^{(1)}-\frac{1}{2}\right)\left(\wp_{1}^{(2)}-\frac{1}{2}\right)+\left(\wp_{2}^{(1)}-\frac{1}{2}\right)\left(\wp_{2}^{(2)}-\frac{1}{2}\right)+\wp_{3}^{(1)} \wp_{3}^{(2)}\right]+\right. \\
& \left.\left(\wp_{2}^{(0)}-\frac{1}{2}\right)\left[\left(\wp_{2}^{(1)}-\frac{1}{2}\right)\left(\wp_{1}^{(2)}-\frac{1}{2}\right)-\left(\wp_{1}^{(1)}-\frac{1}{2}\right)\left(\wp_{2}^{(2)}-\frac{1}{2}\right)\right]\right\} / \sqrt{\wp_{3}^{(1)} \wp_{3}^{(2)}}
\end{aligned}
$$

and $\wp_{i}^{(0)}, \mathrm{i}=1,2,3$ are formal probabilities connected with projector $\widehat{\varrho_{0}}$. The probability addition rule for qutrit (spin-1) can be given analogously. These results provide new formulas for superposition of probabilities which determine the quantum states. Analogous addition rules exist for superposition of probability distributions determining the quantum oscillator states in probability representation of quantum mechanics. Since for any qudit (spin-j or N-level atom) the states are expressed in terms of dichotomic probability distributions describing the statistics of coins the obtained results can be given by statement "God plays coins" replacing the statement "God does not play dice" used in discussion of Einstein and Bohr.

\section{References}

1. S. Mancini, V. I. Man'ko, and P. Tombesi, Phys. Lett. A, 213, 1 (1996)

2. O. V. Man'ko, V. I. Man'ko,. J. Russ. Las. Res., 18, 40 (1997)

3. V. V. Dodonov, and V. I. Man'ko, Phys. Lett. A, 229, 335 (1997)

4. V. I. Man'ko, and O. V. Man'ko, J. Exp. Theor. Phys., 85, 430 (1997)

5. V. N. Chernega, O. V. Man'ko, and V. I. Man'ko,. J. Rus. Las. Res., 39, 128 (2018)

6. V. I. Man'ko, G. Marmo, E. C. G. Sudarshan, F. Zaccaria, J. Phys. A: Math. Gen., 35:33, 7137 (2002)

7. V. N. Chernega, O. V. Man'ko, and V. I. Man'ko,. J. Rus. Las. Res. , 38, 141 (2017) 